\title{
Improvement in structural and electrical properties of cuprous oxide-coated multiwalled carbon nanotubes
}

\author{
SHIVANI DHALL* and NEENA JAGGI \\ Department of Physics, National Institute of Technology, Kurukshetra 136 119, India
}

MS received 23 September 2013

\begin{abstract}
In the present work, cuprous oxide $\left(\mathrm{Cu}_{2} \mathrm{O}\right)$ nanoparticles are coated on multi-walled carbon nanotubes (MWCNTs) using Fehling's reaction. The coating of $\mathrm{Cu}_{2} \mathrm{O}$ nanoparticles on the nanotubes was confirmed by SEM and X-ray diffraction (XRD) spectra. The calculated $I_{D} / I_{G}$ ratio of $\mathrm{Cu}_{2} \mathrm{O}$ (using $3 \% \mathrm{CuSO}_{4}$ by wt)-coated MWCNTs by Raman spectra is found to decrease to 0.94 as compared to 1.14 for pristine MWCNTs. It shows that the presence of $\mathrm{Cu}_{2} \mathrm{O}$ nanoparticles on nanotubes decreases the inherent defects present in the form of some pentagons/heptagons in the honeycomb hexagonal carbon atoms in the structure of graphene sheets of MWCNTs and increases the crystalline nature of MWCNTs, which is also confirmed by the XRD peaks. Whereas the value of $I_{D} / I_{G}$ ratio increases to 1.39 for sample 2 (using $5 \% \mathrm{CuSO}_{4}$ by wt), which represents the structural deformation. Moreover, the electrical conductivity of MWCNTs was increased by 3 times after coating the nanotubes with $\mathrm{Cu}_{2} \mathrm{O}$ (using $3 \% \mathrm{CuSO}_{4}$ by wt).
\end{abstract}

Keywords. Composites; structural studies; electrical properties; lithography.

\section{Introduction}

Recently, there has been rapid progress in the field of nanotechnology by fabricating devices utilizing carbon nanotubes (CNTs) (Iijima 1991; Peng et al 2009; Riehemann et al 2009). The properties of CNTs are keenly modified by the attachment of organic and inorganic compounds such as metals, metal oxides and polymers (Ebbesen 1996; Balasubramanian and Burghard 2005). CNTs have high reactivity and can also easily interact with nanomaterials. This is the main reason behind the enhancement in the structural, electrical, mechanical and sensing properties of CNT composites (Khan and Aziz 2012). Uniform coating of CNTs with nanomaterials has already become a challenging area of research owing to their applications in optoelectronic devices, fuel cells and sensors (Olek et al 2006; Zebli et al 2009; Kim et al 2012; Subramaniam et al 2013). Attachment of $\mathrm{CuO}$, $\mathrm{Cu}_{2} \mathrm{O}, \mathrm{TiO}_{2}, \mathrm{ZnO}, \mathrm{Cu}$-doped $\mathrm{ZnO}$ and $\mathrm{NiO}$ nanoparticles to CNTs has shown great promise for high efficiency in heat transfer applications, electrochemical supercapacitors and gas sensor devices (Reddy et al 2008; Zein and Boccaccini 2008; Liaoa et al 2009; Chen et al 2012). Jha and Ramaprabhu (2008) have decorated MWCNTs with copper nanoparticles by chemical reduction method. They found that thermal conductivity of the composites increased with the concentration of MWCNTs (Jha and Ramaprabhu 2008). Experimental results reveal that

\footnotetext{
*Author for correspondence (shivani.dhall24@gmail.com)
}

$\mathrm{Cu}_{2} \mathrm{O}$-decorated MWCNTs have weak ferromagnetic properties. Xu Long-Shan et al (2007) have studied thermal expansion coefficient of MWCNTs/copper composites. They have observed that the thermal expansion coefficient decreased with increased concentration of MWCNTs in the composites with copper (Xu Long-Shan et al 2007).

In the present work, composites of cuprous oxide nanoparticles with MWCNTs were prepared by chemical route and then enhancement in their structural and electrical properties with respect to pristine MWCNTs investigated using a fabricated device.

\section{Experimental}

\subsection{Synthesis of MWCNTs-based nanocomposites}

The pristine MWCNTs were procured from Global Nanotech (Mumbai, India) with a purity level $>95 \%$. These have been synthesized by CVD method and purified by acids treatment. The process of samples preparation includes three major steps. First, 3\% by wt solution of $\mathrm{CuSO}_{4}$ precursor, called Fehling's solution, with tartaric acid and sodium hydroxide was prepared. A sufficient amount of purified MWCNTs was sonicated in this prepared solution for $1 \mathrm{~h}$ using an ultrasonication bath. Finally, this mixture was stirred at $60{ }^{\circ} \mathrm{C}$ for $1.5 \mathrm{~h}$. During stirring $0.25 \mathrm{~mL}$ formaldehyde as a reducing agent was added drop-wise and then centrifuged. This mixture was washed many times with ethanol and then with distilled water and dried in vacuum. This prepared powder is named as sample 1 . 
The same method was applied for $5 \%$ by wt of $\mathrm{CuSO}_{4}$ solution to obtain sample 2. The chemical reactions involved during the synthesis of cuprous oxide are

$$
\begin{aligned}
& 2 \mathrm{NaOH}+\mathrm{CuSO}_{4} \rightarrow \mathrm{Na}_{2} \mathrm{SO}_{4}+\mathrm{Cu}(\mathrm{OH})_{2}, \\
& \mathrm{Cu}(\mathrm{OH})_{2} \rightarrow \mathrm{CuO}+\mathrm{H}_{2} \mathrm{O}, \\
& \mathrm{CuO}+\text { aldehyde } \rightarrow \mathrm{Cu}_{2} \mathrm{O}+\text { acid. }
\end{aligned}
$$

When MWCNTs were suspended in Fehling's reagent solution and subjected to sonication, the MWCNTs could absorb the copper ions that were complexed with tartrate. Further, by drop-wise addition of formaldehyde into the prepared solution, copper ions $\left(\mathrm{Cu}^{2+}\right)$ were reduced into copper oxide particles on the surface of MWCNTs. In this procedure, MWCNTs acted as templates for the growth of $\mathrm{Cu}_{2} \mathrm{O}$ nanoparticles. The sonication treatment prevented the aggregation of nanoparticles on the nanotubes.

\subsection{Physical characterizations}

XRD spectra of three powder samples were recorded on Rigaku Miniflex using $\mathrm{CuK} \alpha \mathrm{X}$-rays with wavelength $1.54 \AA$. Raman spectra of pristine MWCNTs and prepared samples were recorded on Renishaw using the excitation wavelength $514.5 \mathrm{~nm}$ of $\mathrm{Ar}^{+}$laser in the region $100-4000 \mathrm{~cm}^{-1}$. SEM images of all the samples were taken via Raith Two. The electrical response of the prepared samples was recorded by a Proxima probe set up. For carrying out these studies, both samples were dispersed in $\mathrm{N}, \mathrm{N}$-dimethyl formamide (DMF) by taking $0.2 \mathrm{mg}(2 \%$ by wt) of each sample in $10 \mathrm{~mL}$ solution of $\mathrm{DMF}$.

\subsection{Device fabrication}

For sensor fabrication, first, a pattern of interdigitated electrodes (IDEs) was made using Klevin software with finger width $10 \mu \mathrm{m}$, finger length $100 \mu \mathrm{m}$ and a gap between the two fingers being $5 \mu \mathrm{m}$. A thin layer of $\mathrm{SiO}_{2}$ of thickness $120 \mathrm{~nm}$ was deposited on Si wafer by dry oxidation. The designed mask has been prepared by a laser writer and was transferred on to the prepared wafer using photolithography technique (double side alignerEVG 620). A chromium-gold layer of thickness $100 \mathrm{~nm}$ $(\mathrm{Cr}=20 \mathrm{~nm}, \mathrm{Au}=80 \mathrm{~nm})$ was deposited on the developed substrate by a thermal evaporator. Finally, $\mathrm{Cr}-\mathrm{Au}$ was removed by wet etching to get the desired device. The optical image of fabricated IDEs is shown in figure 1 . Then thin films of synthesized composites were dropcasted on IDEs using a micropipette. The solvent was removed by heating the device, retaining only a network of composites in between the electrodes.

\section{Results and discussion}

As shown in figure 2(a), XRD spectra of P-MWCNTs show a broad diffraction peak at $2 \theta=25 \cdot 5^{\circ}$ corresponding

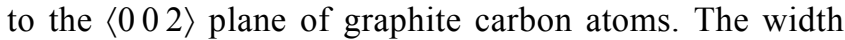
and position of the diffraction peak $\left(\begin{array}{lll}0 & 0 & 2\end{array}\right)$ are related to the structural ordering of the nanotubes (Gupta and Farmer 2011). The same diffraction peak appears at $2 \theta=26 \cdot 2^{\circ}$ for $\mathrm{Cu}_{2} \mathrm{O}$-coated nanotubes (samples 1 and 2 ) as shown in figure $2(\mathrm{~b}-\mathrm{c})$. Due to strong interaction between nanotubes and $\mathrm{Cu}_{2} \mathrm{O}$ nanoparticles, a significant shift towards higher angle occurred (figure $2 b-c$ ) which resulted in a compressive stress and hence interplanar spacing of the tubes is changed (Hee et al 2002; Zhi et al 2006). Moreover, the calculated average $d$-spacing value using Bragg's law for $\mathrm{Cu}_{2} \mathrm{O}$-coated nanotubes is found to increase to $3.39 \AA$ as compared to $3.47 \AA$ for pristine nanotubes. The other diffraction angles at $2 \theta=29.8$, $36 \cdot 7,38 \cdot 1,42 \cdot 7,44 \cdot 3,61 \cdot 6$ and $77 \cdot 6^{\circ}$ in figure $2(\mathrm{~b}-\mathrm{c})$ are

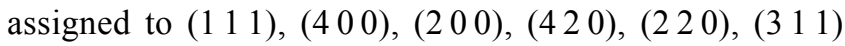
and (222) planes for the cubic structure of $\mathrm{Cu}_{2} \mathrm{O}$ by comparing the results with reported JCPS data card no. 050667 and 011142 . The average calculated crystallite size of $\mathrm{Cu}_{2} \mathrm{O}$ particles in samples 1 and 2 were found to be 10 and $15 \mathrm{~nm}$. The lower crystallite size always corresponds to lower loading of nanoparticles and the higher crystallite size to higher metal loading or vice versa (Randeniya et al 2010).

Raman spectroscopy is a powerful technique used to characterize carbon-based nanomaterials, like fullerenes and CNTs. Raman spectra (figure 3a) show the presence of three bands, viz. D-band at $1361 \mathrm{~cm}^{-1}$, G-band at $1595 \mathrm{~cm}^{-1}$ and $\mathrm{G}^{\prime}$-band at $2700 \mathrm{~cm}^{-1}$ for the pristine MWCNTs.

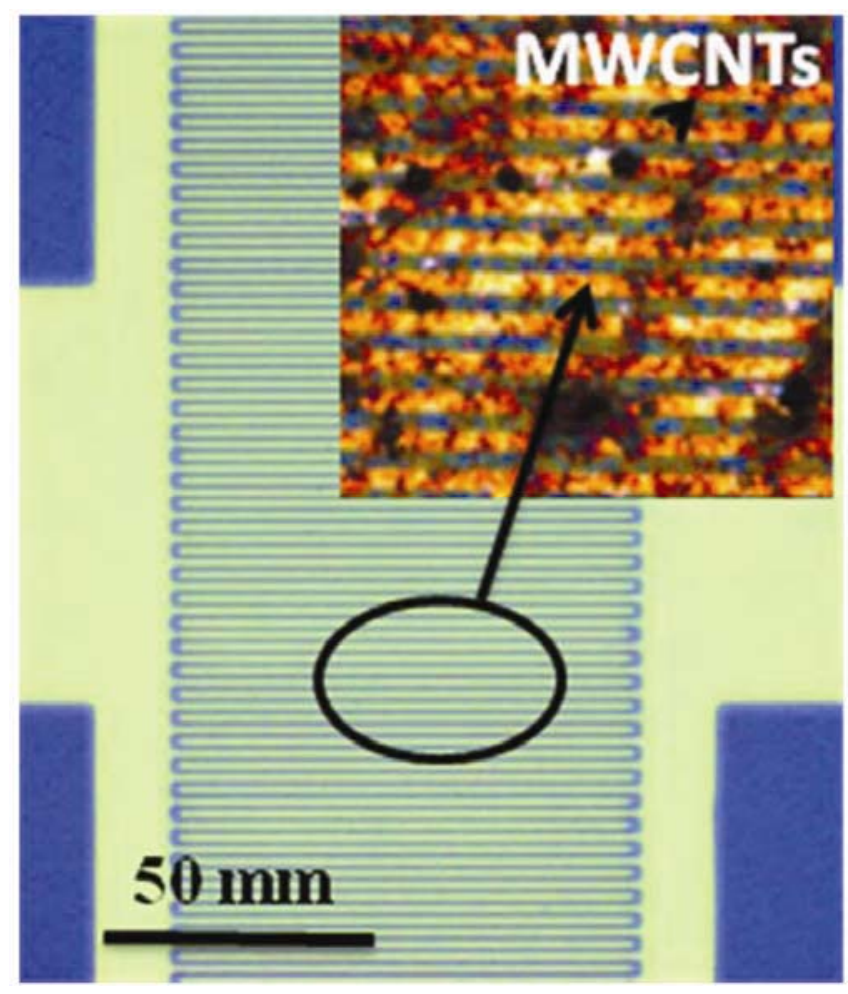

Figure 1. Optical image of a fabricated device. 


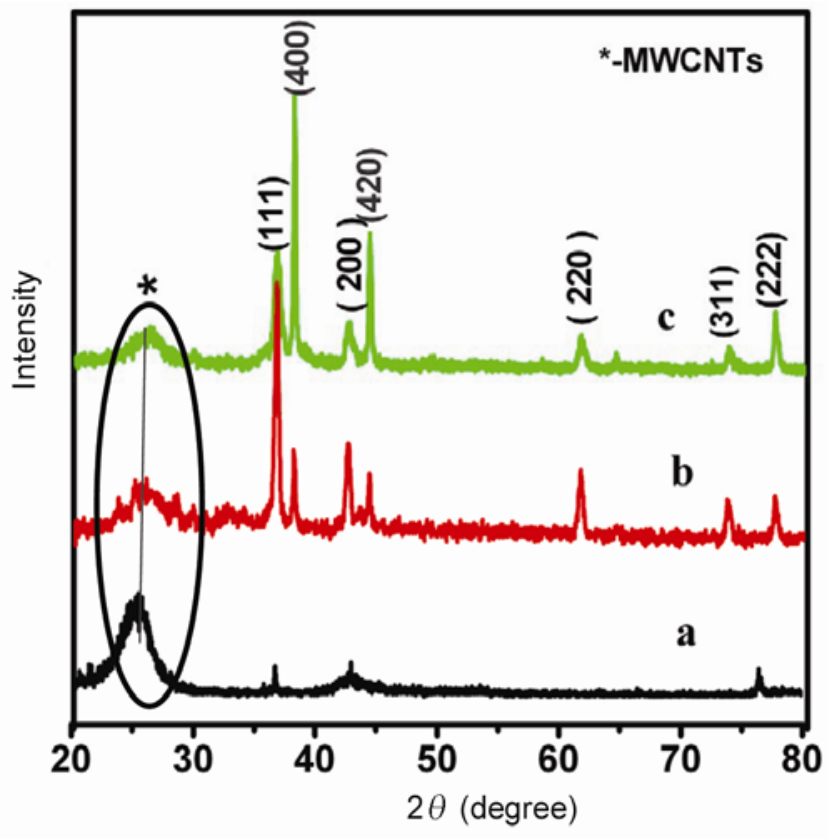

Figure 2. XRD spectra of (a) pristine MWCNTs, (b) sample 1 and (c) sample 2 .

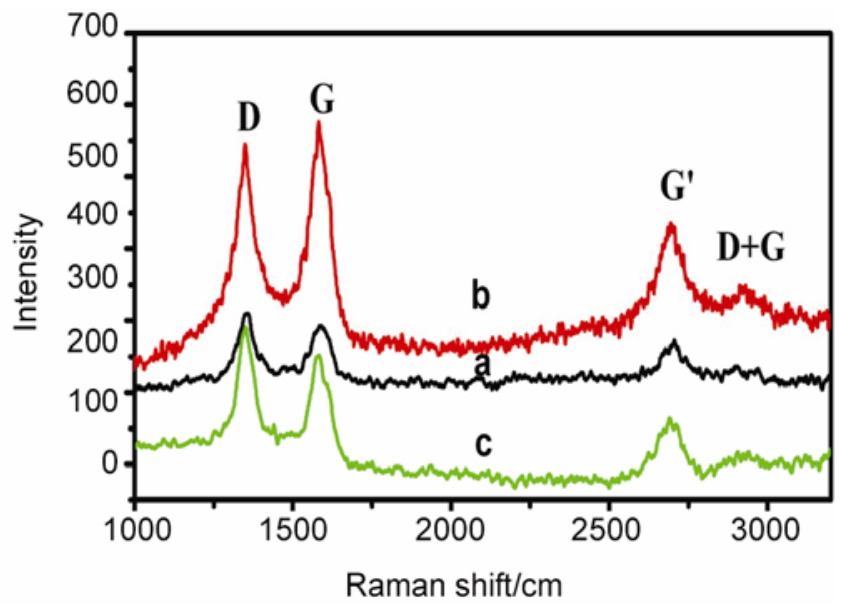

Figure 3. Raman spectra of (a) pristine MWCNTs, (b) sample 1 and (c) sample 2.

Moreover, D-band indicates the presence of the disordered carbon structure of nanotubes and is related with breathing mode of $s p^{3}$ sites present in carbon tubes in the form of rings. G-band, known as graphitic mode, is observed due to stretching vibration of any pair of $s p^{2}$ sites (Bokobza 2009; Tessonnier et al 2009; Gallegos et al 2011). The intensity ratio of D-band to G-band $\left(I_{\mathrm{D}} / I_{\mathrm{G}}\right)$ is related to degree of disorder of the nanotubes and inversely proportional to the crystalline nature of CNTs (Shan et al 2007; Gallegos et al 2011). As shown in figures $3(\mathrm{~b}-\mathrm{c})$, these characteristic peaks are still present in the Raman spectra of $\mathrm{Cu}_{2} \mathrm{O}$-coated nanotubes.
Table 1. Raman analysis of all three samples.

\begin{tabular}{lccccc}
\hline Sample name & D band & G band & $\mathrm{G}^{\prime}$ band & $I_{\mathrm{D}} / I_{\mathrm{G}}$ & $L_{\mathrm{a}}(\mathrm{nm})$ \\
\hline Pristine MWCNTs & 1361 & 1596 & 2702 & $1 \cdot 14$ & $14 \cdot 6$ \\
Sample 1 & 1350 & 1588 & 2695 & $0 \cdot 94$ & $17 \cdot 7$ \\
Sample 2 & 1347 & 1576 & 2692 & $1 \cdot 39$ & $11 \cdot 9$ \\
\hline
\end{tabular}

Therefore, the inherent structure of the pristine tubes is retained after coating of nanoparticles. The calculated value of $I_{\mathrm{D}} / I_{\mathrm{G}}$ ratio for pristine MWCNTs is $1 \cdot 14$. For sample 1 , the value of intensity ratio is decreased to 0.94 . It means that attachment of $\mathrm{Cu}_{2} \mathrm{O}$ nanoparticles on MWCNTs have decreased the inherent presence of defects in the form of some pentagons or heptagons in the honeycomb structure of graphene sheets in MWCNTs and increased the crystalline order of MWCNTs. The calculated value of $I_{\mathrm{D}} / I_{\mathrm{G}}$ for sample 2 has been increased to 1.39. The increased value of $I_{\mathrm{D}} / I_{\mathrm{G}}$ for sample 2 can be due to the introduction of functional groups (discussed in FT-IR) and also crystallinity of MWCNTs is decreased. The insertion of functional groups on the nanotubes walls is interpreted as defects in the structure (Tessonnier et al 2009). The intensity of $\mathrm{G}^{\prime}$-band is increased (figure $3 \mathrm{~b}$ ) to a high value for sample 1 as compared to sample 2 . The intensity of this peak depends strongly on the metallicity of the nanotubes, which means that sample 1 contains high electrical properties as compared to sample 2 . In addition, the Raman spectra of $\mathrm{Cu}_{2} \mathrm{O}$ nanoparticles attached nanotubes contain new band at 2932 (figure 3b) and $2925 \mathrm{~cm}^{-1}$ (figure $3 \mathrm{c}$ ), which is the combination of D and $\mathrm{G}$ band. The appearance of this combination band also confirms the attachment of $\mathrm{Cu}_{2} \mathrm{O}$ nanoparticles, giving rise to enhancement of the Raman combination band due to resonance effect. We have also calculated the crystallite size $\left(L_{\mathrm{a}}\right)$ from the following formula (Cancado et al 2006)

$$
L_{\mathrm{a}}(\mathrm{nm})=\frac{560}{E_{\mathrm{L}}^{4}}\left(\frac{I_{\mathrm{D}}}{I_{\mathrm{G}}}\right)^{-1} .
$$

The crystallite size of sample 1 has been increased to $17.7 \mathrm{~nm}$ as compared to $11.9 \mathrm{~nm}$ size of sample 2. The detailed Raman analyses of all samples are shown in table 1 .

Figure 4(a-c) shows SEM images of pristine MWCNTs and the prepared samples. SEM images of figures $4(b-c)$ clearly indicate that there is an interface between MWCNTs and $\mathrm{Cu}_{2} \mathrm{O}$ nanoparticles. The good bonding between MWCNTs and $\mathrm{Cu}_{2} \mathrm{O}$ and the presence of a network structure of MWCNTs at intergranular positions is also clearly depicted in SEM images.

FT-IR spectra of pristine MWCNTs are shown in inset of figure 5 . The spectra of sample 1 contain a broad peak centred at $3412 \mathrm{~cm}^{-1}$ corresponding to $\mathrm{O}-\mathrm{H}$ stretching vibration. This same peak in sample 2 appears at 3435 


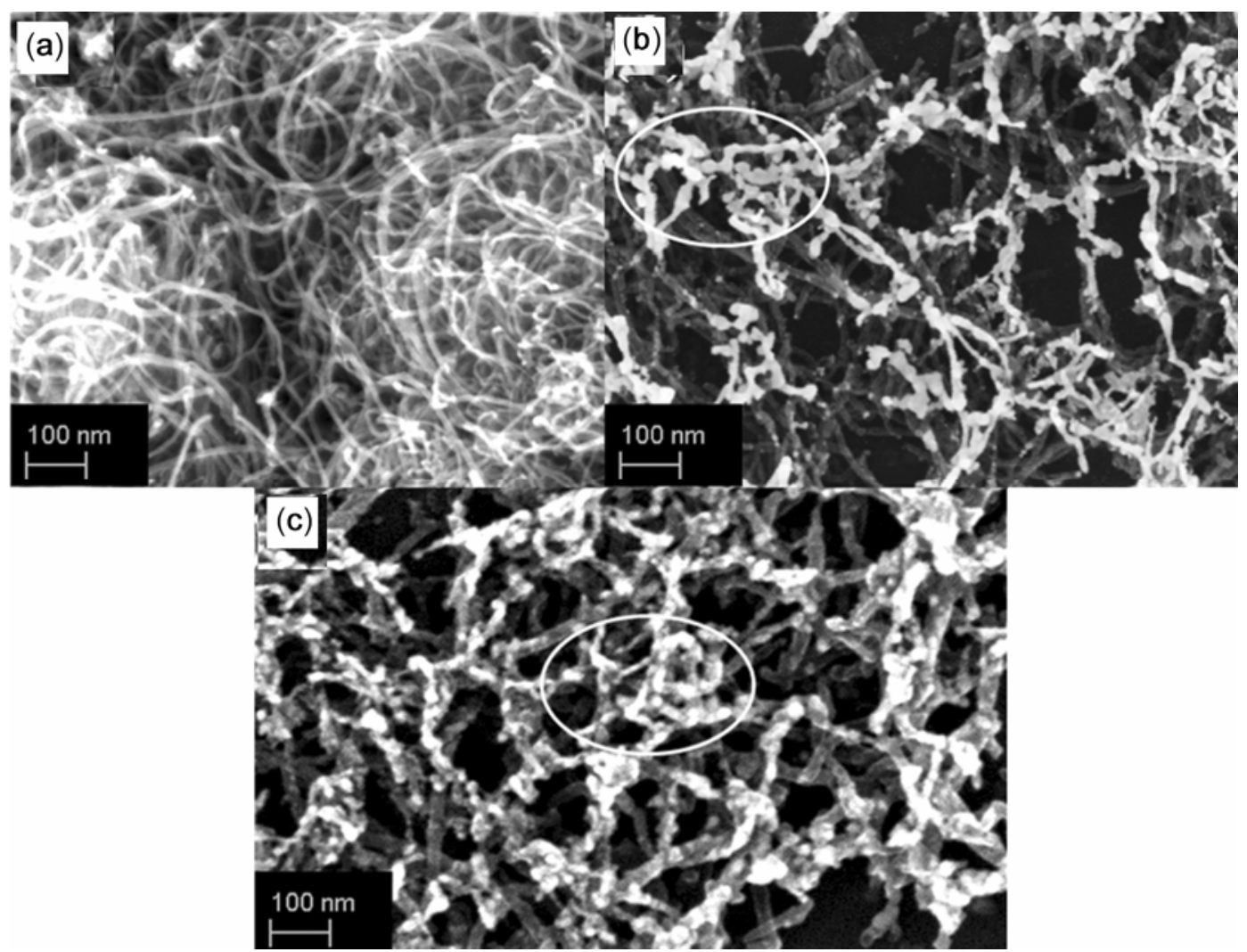

Figure 4. SEM image of (a) pristine MWCNTs, (b) sample 1 and (c) sample 2.

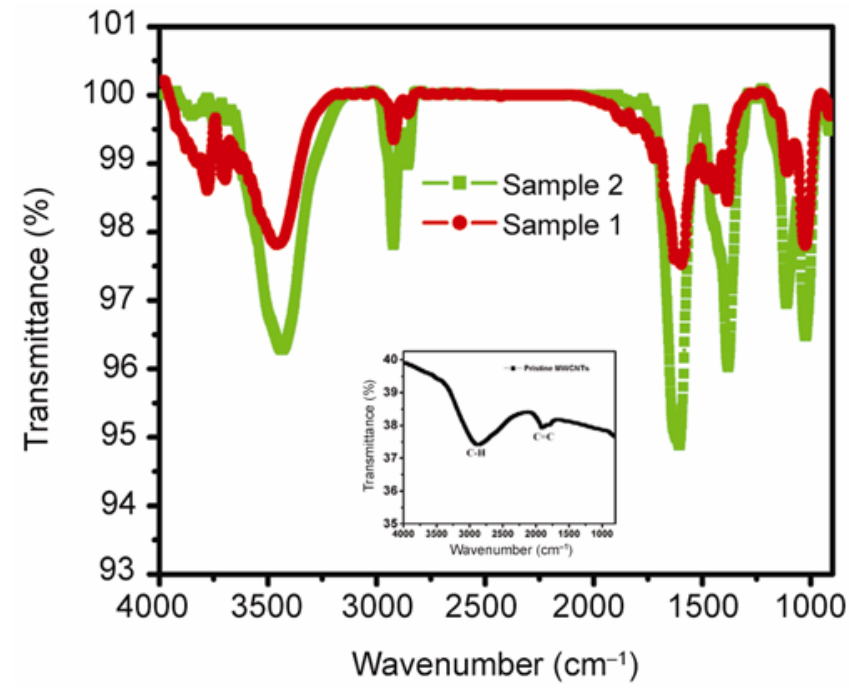

Figure 5. FT-IR spectra of sample 1 and sample 2.

and $3461 \mathrm{~cm}^{-1}$. Some peaks are absent in sample 1 , but present in sample 2, viz. 1025 and $1109 \mathrm{~cm}^{-1}$ corresponding to $\mathrm{C}=\mathrm{O}$ stretching vibration, 1437 and $1484 \mathrm{~cm}^{-1}$ related to $\mathrm{C}-\mathrm{H}, 1597 \mathrm{~cm}^{-1}$ for $\mathrm{C}=\mathrm{C}$ stretching vibration and $1720 \mathrm{~cm}^{-1}$ related to $\mathrm{C}=\mathrm{O}$ stretching vibration. It is clear that, when the concentration of $\mathrm{CuSO}_{4}$ precursor is increased, more and more functional groups are attached on to the MWCNTs walls.

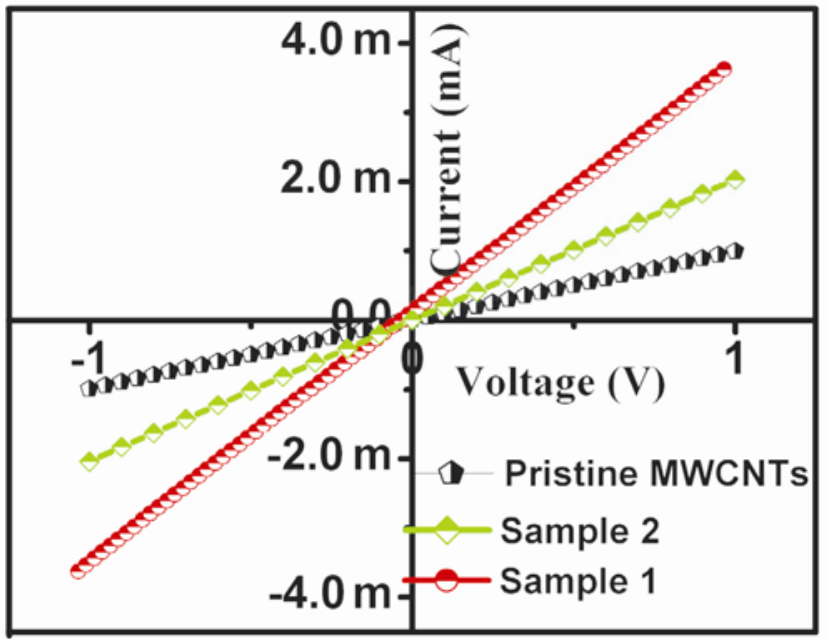

Figure 6. $I-V$ characteristics of all three samples.

The electronic transport properties of pristine and $\mathrm{Cu}_{2} \mathrm{O}$-coated tubes were investigated by depositing thin films of these samples on the top surface of IDEs. The variation in current-voltage characteristics of three samples are shown in figure 6 . The contact improvement between the tubes and electrodes was avoided by annealing the device at $100^{\circ} \mathrm{C}$ before drop casting the films on the top surface. $I-V$ characteristics for sample 1 show higher 

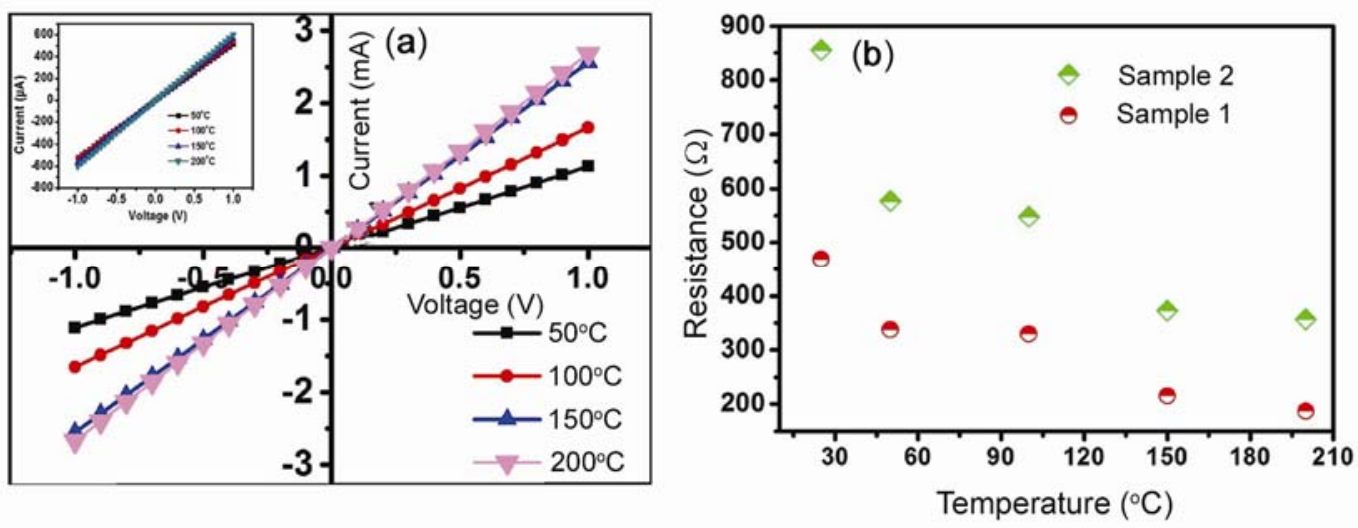

Figure 7. (a) Temperature-dependent $I-V$ characteristics of sample 2 and (b) variation in resistances with the temperature.

conductivity as compared to sample 2 and pristine tubes. These results agree with the analysis of Raman spectroscopy as discussed earlier. Also, the linear $I-V$ curve indicates the ohmic contacts between $\mathrm{Cu}_{2} \mathrm{O}$ nanoparticles and nanotubes. Figure 7 shows temperature-dependent $I-V$ characteristics of sample 2. The inset of this figure presents the $I-V$ characteristics of pristine tubes. The increase in the current or the decrease in the resistance (figure $7 \mathrm{~b}$ ) observed with increasing temperature indicates the semiconducting behaviour of $\mathrm{Cu}_{2} \mathrm{O}$-coated nanotubes. Similar type of behaviour has been reported by Hoa et al (2010) in their work.

\section{Conclusions}

The effect of precursor concentration on the structural and electrical properties of pristine MWCNTs is studied in the present work. Raman spectroscopy and SEM have been utilized to ascertain the composite formation and also its analysis led to the conclusion that sample 1 contains good crystalline and conductive nature of the nanotubes. Owing to its improved electrical properties, the present route provides a simple approach to synthesize nanotubes-based nanocomposites, which finds application in gas sensor and semiconducting technology.

\section{Acknowledgements}

The authors acknowledge the support and help for fabrication of the device and electrical measurements carried out at the Centre of Excellence in Nanoelectronics (CEN) under Indian Nanoelectronics Users' Programme at Indian Institute of Technology (IIT), Bombay, which has been sponsored by the Department of Information Technology (DIT), Government of India.

\section{References}

Balasubramanian K and Burghard M 2005 Small 1180

Bokobza L 2009 Vib. Spect. 5152

Cancado L G, Takai K, Enoki T, Endo M and Kim Y A 2006 Appl. Phys. Lett. 88163106

Chen C S, Xie X D, Liu T G, Lin L W, Kuang J C, Xie X L, Lu L J and Cao S Y 2012 J. Nanopart. Res. 14817

Ebbesen T W 1996 Adv. Mater. 8155

Gallegos A K C, Frausto C, Frade L A O and Orozco G 2011 Vib. Spect. 5749

Gupta S and Farmer J 2011 Appl. Phys. 109014314

Hee S W, Seung B Y, Jeunghee P, Hyunik Y, Kwang P S and Sangsig K J 2002 Chem. Phys. 1169492

Hoa N D, Quy N V, Jung H, Kin D, Kim H and Hong S K 2010 Sens. Actuators 146266

Iijima S 1991 Nature 35456

Kim D W, Rhee K Y and Park S J 2012 J. Alloys Compd. 5306

Khan N A and Aziz S 2012 J. Alloys Compd. 538183

Liaoa X N, Hub C Y, Wanga Q L and Lid F Y 2009 J. Chinese Chem. Soc. 56475

Jha N and Ramaprabhu S 2008 J. Phys. Chem. C112 9315

Olek M, Busgen T, Hilgendorff and Giersig M 2006 J. Phys. Chem. B110 12901

Peng X, Misewich J A and Wong S S 2009 Chem. Soc. Rev. 381076

Randeniya L K, Bendavid A, Martin P J and Tran C 2010 Small 61806

Reddy K R, Sin B C, Yoo C H, Park W, Ryu K S, Lee J S, Sohn D and Lee Y 2008 Scr. Mater. 581010

Riehemann K, Schneider S W, Luger T A, Godin B, Ferrari M and Fuchs H 2009 Angew. Chem. Int. Ed. 48872

Subramaniam C, Yamada T, Kobashi K, Sekiguchi A, Futaba D N, Yumura M and Hata K 2013 Nature 42202

Shan X L et al 2007 Trans. Nonferrous Met. Soc. 171065

Tessonnier J P et al 2009 Carbon 471779

Zebli B, Vieyra H A, Carmeli I, Hartschuh A, Kotthaus J P and Holleitner A W 2009 Phys. Rev. B79 205402

Zein S H S and Boccaccini A R 2008 Ind. Eng. Chem. Res. 476598

Zhi C, Bando Y, Tang C and Golberg D 2006 J. Phys. Chem. B110 8548 